\title{
Impact damage detection on composites using optical NDT techniques
}

\author{
Jasson Gryzagoridis, Dirk Findeis \\ Mechanical Engineering Department \\ University of Cape Town, South Africa. \\ Phone: +27216503229 \\ Fax: +27216503240 \\ E-mail: jasson.gryzagoridis@uct.ac.za
}

\begin{abstract}
The paper presents the work that was carried out in the detection and, beyond of what is obviously visible to the naked eye, the extent of impact damage inflicted on composite panels designed and manufactured for structural applications in the aerospace industry. Using a drop-weight impact tester the composite specimens were subjected to controlled impact conditions i.e. relatively low velocity impact levels analogous to those expected from the drop of a heavy tool, collision with a bird etc. The composite specimens consisted of Monex core sandwiched between thin carbon/glass fibre reinforced plastic skins, typically identical to those used in the fabrication of unmanned air vehicles and helicopter rotor blades. The investigation attempted to establish the probability of detecting such barely visible impact damage with optical non-destructive testing methods such as Digital Shearography, Electronic Speckle Pattern Interferometry and Infrared Thermography. These NDT techniques were chosen to be employed in this study, because they are non-contacting, whole field, not limited to particular material types, provide fast and easy results in real-time and have been proven reliable in a large number of applications in the laboratory/factory/field environment.
\end{abstract}

KEYWORDS: Shearography, ESPI, Infrared Thermography, composite materials, laser based NDT, optical, speckle images, thermo grams, barely visible impact damage.

\section{Introduction}

The usage of composite materials in industries relating to aerospace, automotive, weapons/aircraft manufacture etc. is ever increasing in an exponential manner which is rightly justified by the fact that their mechanical properties are adequately meeting engineering requirements and particularly their specific strength (strength to density value), a property which is often referred to as strength-to-weight ratio. However the mechanical properties of composites may degrade severely due to defects and flaws arising from damage sustained for example through impact. This damage may not be visible to the naked eye and may result to the failure of a system, after repeated cycles or time of operation. It is known that impact causes the core or matrix in composites to crack in a characteristic way, known as star cracks, which are internal and difficult to detect when using traditional non destructive testing techniques. "It stands to reason that 
impact damage, no matter how small, should be detected as soon as possible in order to monitor its growth during use of the component or structure". ${ }^{(1)}$ To this end it is desirable to employ non destructive testing techniques such as Digital Shearography, ESPI and Thermography, all known for their high sensitivity. As it is evidenced in the literature, these techniques have been continuously providing examples of success in the detection of defects among the wide range of types of flaws found in composites such as de-laminations, cracks, inclusions, voids, broken filaments/mats etc. In a recent presentation Dave Galela of the FAA Hughes Technical Center ${ }^{(2)}$ gave comparisons of the probability of detection of a defect using various NDT techniques. Forming part of a cluster of advanced NDT techniques both shearography and thermography performed very well.

\subsection{Digital Shearography}

Digital Shearography has its origins firstly as a strain measurement technique (1974) and later as a non-destructive testing tool (1982) as reported by Hung ${ }^{(3,4)}$. Digital Shearography as a non-contacting, whole filed view and practically real time technique, is capable of revealing sub surface defects as a localized disturbance in the fringe pattern depicting the gradient of out-of-plane surface displacements on the component under test. In response to a mild perturbation of the specimen under test by mechanical, thermal, pressure or vibratory means the surface of the test piece which is illuminated with a laser changes its speckled image. The subtraction of two speckled images of the object's surface, obtained one before and the other after the perturbation, results in the fringe pattern which resembles zebra stripes superimposed on the object's image. "The anomalies in the fringe pattern, basically display the position and approximate size of the defect, however not its depth relative to the surface"(5). Typical Digital Shearographic NDT set-ups, similar to the one depicted in figure 1(left), include a personal computer equipped with software to process the images of the object under test. A single wavelength light source (a laser) is used to illuminate the object thus producing a unique speckled image. The images are obtained via a digital camera viewing the object through some shearing optics and are stored in the pc's image digitizer. A typical shearing device (favoured by the authors as it is used in their portable shearography system ${ }^{(4)}$ ), is the modified Michelson interferometer as illustrated in figure 1(right).
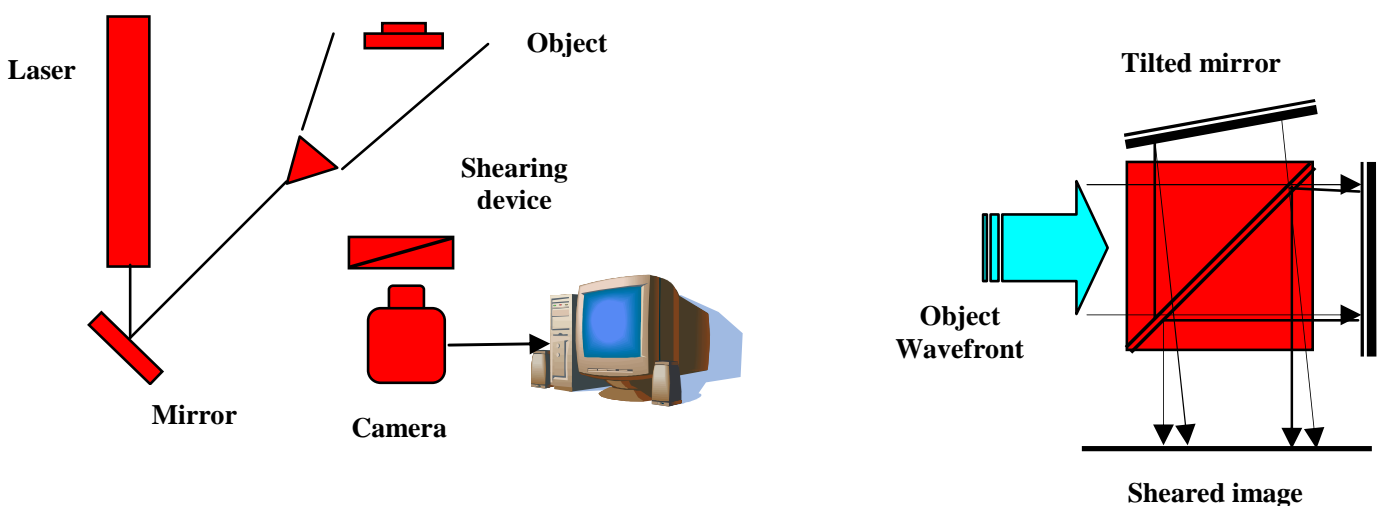

Figure 1. Typical Shearography set-up (left) and image shearing device (right) 
It can be shown that measurements of the gradient of the normal displacement of points on a given surface (along a given direction) can be effected using the following equation

$$
\frac{\partial \delta}{\partial x}=\frac{\lambda N}{2 S}
$$

where $\frac{\partial \delta}{\partial x}$ is the displacement gradient along the $\mathrm{x}$ axis (in this case or $\mathrm{y}$ axis or any chosen direction), $\lambda$ is the wave length of the laser, $N$ is the number of fringes observed, and $S$ is the lateral shear imposed by the image shearing device.

\section{$1.2 \quad E S P I$}

Electronic Speckle Pattern Interferometry (ESPI) was first announced or demonstrated by Butters \& Leendertz ${ }^{(6)}$ as a special version of holographic interferometry where the display of a fringe pattern is upon a pc's monitor, practically in real time. The fringe pattern is interpreted as a contour map of the surface displacement of an object when it is mildly stressed and compared to a reference state, identical to the process followed in the technique of Digital Shearography described above. The displacement can be measured either in the in-plane or out-of-plane direction depending on the arrangement of the optical components. The out-of-plane displacement sensitive arrangement, as shown in figure 2, is the more versatile of the two as it makes better usage of the light originating at the laser. The set-up is very similar to the Shearography one shown in figure 1 (left), with the exception of the reference beam created by the beam splitter and the two laser beam steering mirrors. The reference beam is combined with the object reflection beam behind the camera lens producing a unique speckled image. The process of obtaining a fringe pattern (as a contour of the normal surface displacements) superimposed on the image of the object's surface under test is identical to that detailed for the shearography technique. It can be shown that the out of plane displacement of any point on the object can be calculated using the following equation

$$
\delta=\frac{\lambda * \mathrm{~N}}{2}
$$

Where $\delta$ is the surface's normal displacement at a particular point in the area viewed, $\lambda$ is the laser wavelength and $N$ the number of fringes observed covering the area from a reference zero displacement up to the point in question. Equation 2 could also be used to estimate the sensitivity of the technique, where for a single fringe increment the order of magnitude of the normal displacement observed (for a HeNe laser) is approximately 330 nanometres.

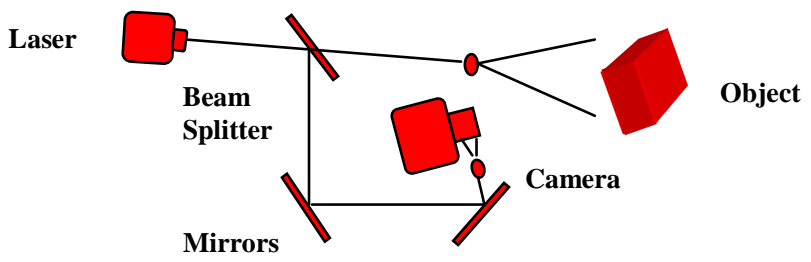

Figure 2. Typical ESPI set-up for out-of-plane displacement measurement 
The Shearographic "head" featuring the Michelson type shearing apparatus is also capable of obtaining ESPI interferograms as has been already reported by Gryzagoridis and Findeis ${ }^{(8)}$ with the relevant passage briefly described in the section below.

\subsection{Dual-function, (Shearography/ESPI)}

When one compares the set-ups of Digital Shearography and ESPI (figures 1 and 2 respectively) the similarity is apparent, with the major difference being the reference beam. For example the reference beam in the Shearography set-up is generated by the actual surface of the test piece and therefore it carries the signals of the body motion. This of course is highly desirable in Shearography because it reduces the effect of environmental disturbances that de-correlate the speckle images. In the ESPI set-up the reference beam is produced from the original illumination beam and without being disturbed is combined with the beam reflected from the object by a beam splitter located after the camera's lens. It appears therefore that if a reference beam can be introduced in the shearography set-up and combined with the image of the object under test we would obtain an ESPI result. This is relatively easily accomplished by introducing in say one third of the illumination field of the object an optically diffuse flat surface. The surface will reflect its image onto the beam splitter where it would combine with the reflected image of the surface under test and create the fringe pattern that exhibits displacements normal to the surface. What has been described above is depicted in figure 3 where we note that the shear distance is substantially increased (by the tilting of one of the mirrors) to and beyond the point that the two images share no common portion. Under these conditions with the reference beam generated by the neutral surface, one image (the lower one in fig. 3 or the one transmitted through the beam splitter) of the portion of the surface under test is combined with the reference beam creating ESPI conditions.

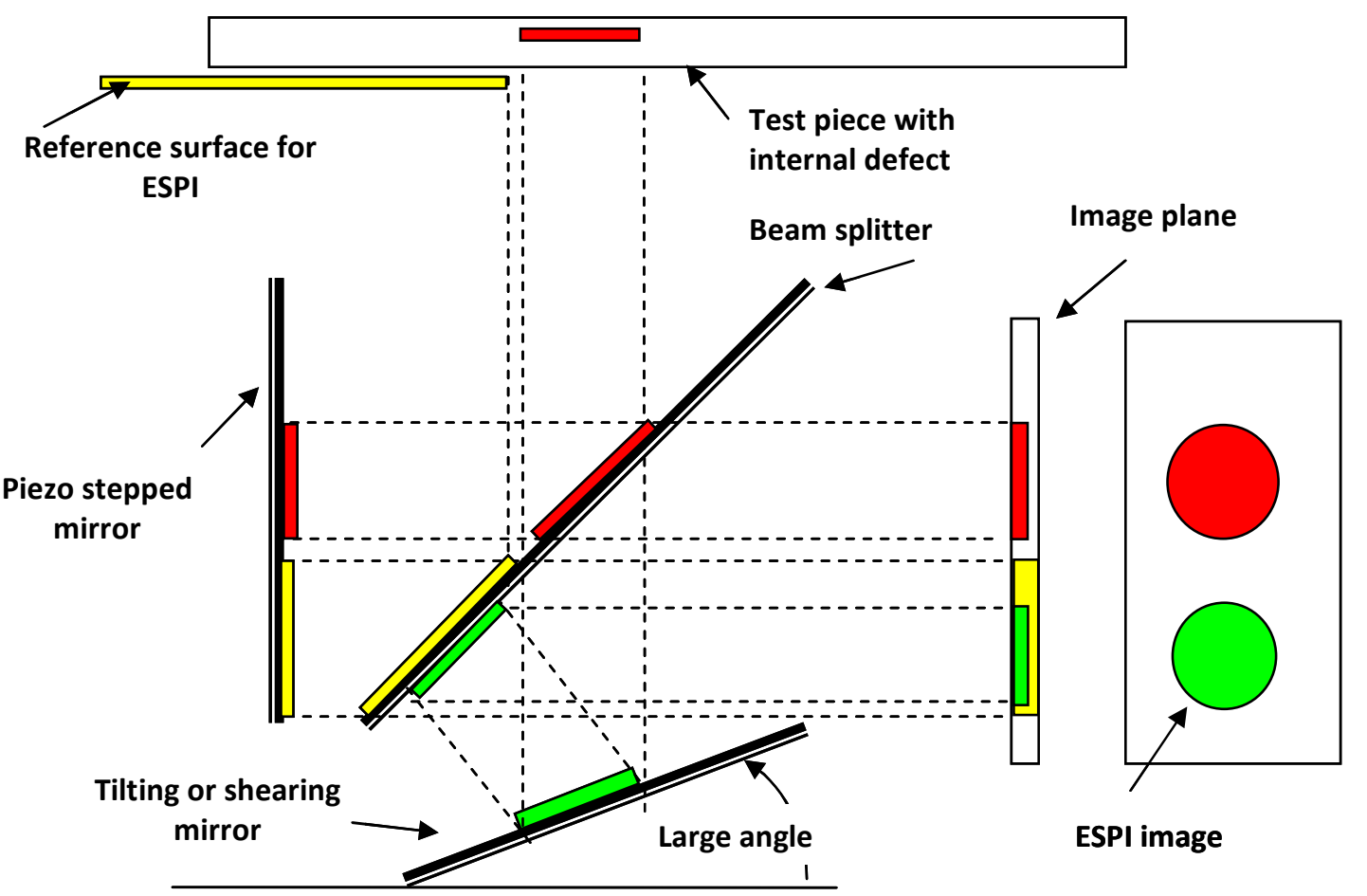

Figure 3. Transformation of Shearography to ESPI 


\subsection{Infrared Thermography}

Thermography is another non-contacting, full view NDT technique which also operates in real time producing results known as thermograms, which are typically depicted by colour bands representing contours of equal temperature generated after applying heat to the test surface. There is ample evidence, in the relevant literature, of the testing of aerospace components/structures using Thermographic cameras that detect infrared radiation emitted from any warm surface. If there is a subsurface defect the tendency will be for the region of the material surrounding the defect to be warmer since the defect impedes the transfer of heat and therefore it will appear as a bright spot against the cooler material, normally depicted as dark background. "A given surface radiates an amount of thermal energy at a given temperature as a function of its emissivity, a property that relates this amount to the idealized maximum that a "black body" is capable of emitting. Since variations of infrared intensity are related to surface temperatures it is imperative that the surface under test possesses uniform emissivity" 7 .

\section{Experimental procedure and results}

The experiments were conducted in the NDT laboratory of the Mechanical Engineering Department at the University of Cape Town using our proprietary Shearography/ESPI apparatus which is also equipped to perform the phase stepping technique. Therefore in essence UCT's dual purpose optical NDT system was used to detect impact damage both in Shearographic and ESPI formats, while a relatively very inexpensive Thermographic camera known as IRISYS-1011 Universal Thermal Imager was employed to obtain thermograms of the same impact damage on the composite specimens. The test pieces utilized in this study were prepared from the wing of an unmanned aerial vehicle (UAV) and the main rotor blade of an Oryx helicopter. The defects amounted to crushed honeycomb core underneath the skin of the specimens which barely showed any marks of the impact.
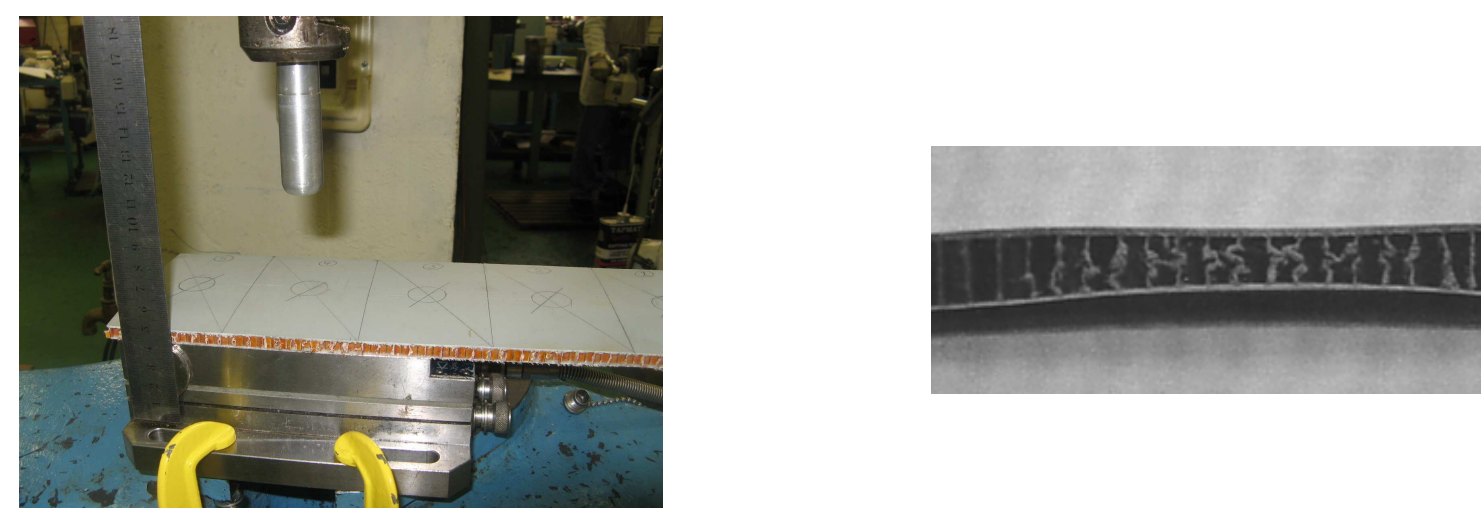

Figure 4. Left, setup used to create the impact damage on the specimen with typical core damage obtained as depicted in the image to the right.

Figure 4 shows a steel rule vertically positioned to measure the heights from which impact damage was to be initiated. The impactor head was hemispherical, made from an 
aluminium bar $20 \mathrm{~mm}$ in diameter which was guided to fall on the specimen that rested on the tool dynamometer. The output (Newtons) of the tool dynamometer was read off an oscilloscope together with the impact time. Thus the experimental impulse could be found and compared to the theoretical impulse values however, for the purpose here being a comparative exercise, we will not require these calculations and results. The defects were created accordingly by releasing the impactor from different heights. Care was taken to ensure that the impactor was released from heights that did not result in it creating visible damage to the surface of the specimen. (cracks, indentations, scratches etc.) Typical phased stepped shearographic results obtained by mildly heating the specimen are depicted in figure 5 below; impacts for this specimen were accomplished from heights of 200,150 and $100 \mathrm{~mm}$ respectively starting from the left of the image.

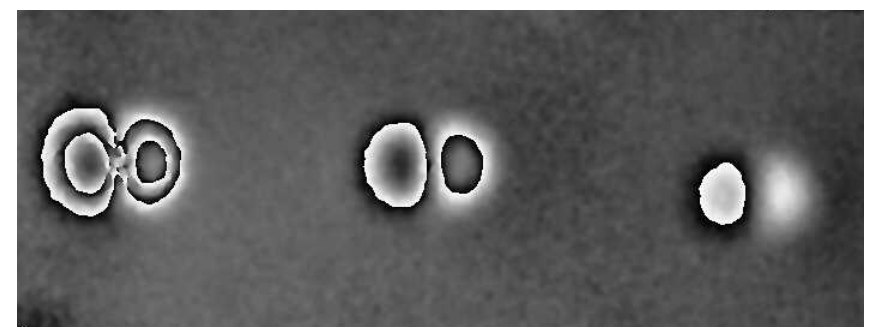

Figure 5. Internal impact damage revealed with Shearography

The same specimen as depicted in figure 5 was examined using the IRISYS-1011 Universal Thermal Imager after the specimen was heated mildly using a hair dryer for a few seconds. The results as depicted in figure 6 , were obtained with a little effort by continuously manipulating the sensitivity of the infrared camera, are comparable to those obtained with the shearographic system.

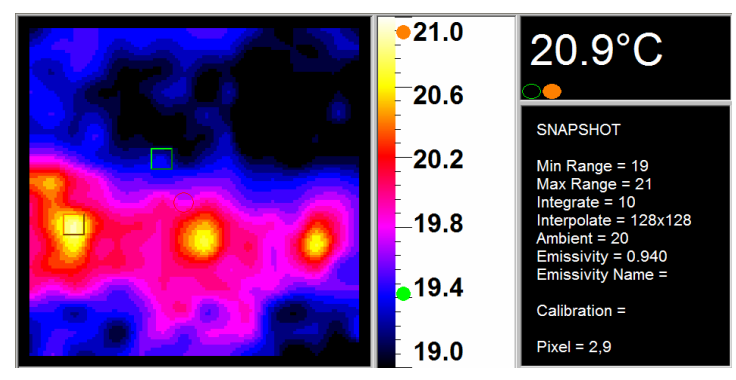

Figure 6. Internal impact damage revealed with Thermography

Continuing with the same specimen and concentrating on the middle of the three impacts that were simulated on the UAV's wing composite specimen, the position was examined for signs of internal damage by using UCT's Shearography system in the ESPI mode. The result which is shown in figure 7 below is as dramatic as those obtained in the Shearographic mode (see figure 5 above). The image on the left for both assembles is in ESPI format, the first as an intensity image and the second as a filtered stepped phase interferogram and the ones on their right are their respective Shearography counterparts. The fringes that appear at an angle in the ESPI images are indication of body motion to which ESPI is very susceptible. They were probably 
caused by the motion of the specimen itself, rather than motion of the reference surface that was placed quite securely and rigidly in front of the test piece.
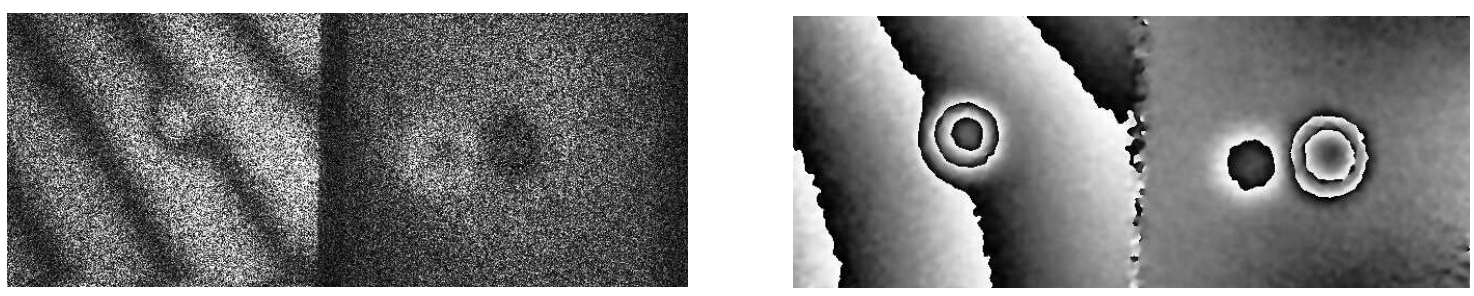

Figure 7. Internal impact damage (middle of the three) revealed with ESPI intensity image on the left and filtered stepped phase image on the right

Finally the tests indicated that at all times Shearography continued to reveal the internal damage due to impact for a longer period compared to the Thermographic technique. This is demonstrated in the images depicted in figure 8 below.
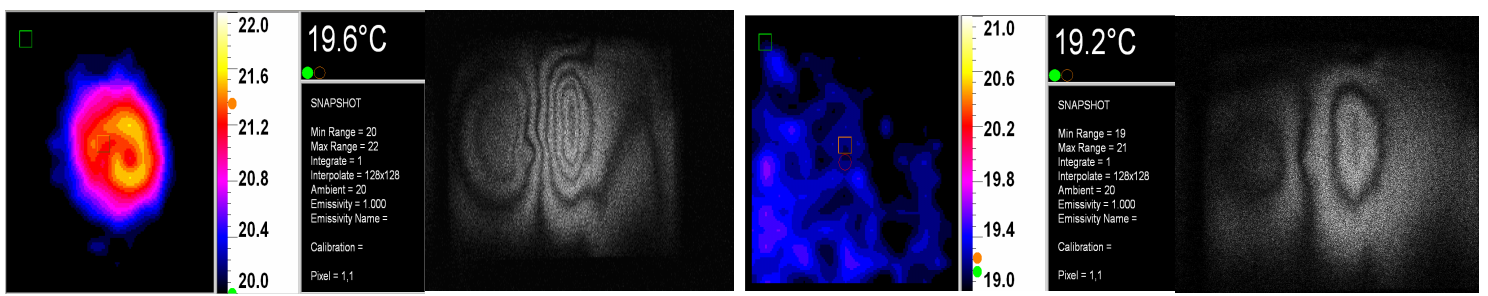

Figure 8. The Shearographic system continued to indicate the presence of a defect long after the Thermographic technique

\section{Conclusions}

The aim of this work was to re-affirm the suitability of optical NDT techniques such as the laser based Shearography and ESPI as well as Infrared Thermography in detecting barely surface visible, impact damage on aerospace composites. The results support previous claims in the relevant literature ${ }^{(2)}$ that these techniques do in fact perform very well during NDT testing of specimen types, modeled after a range of construction found on aircraft; carbon graphite and fiberglass skin over Nomex core for various flaw types including impact damage. It was possible to produce internal damage to the honeycomb core without any visible signs on the skin covering the core by judiciously performing the impacts from "safe" heights. It became evident, as was expected, that the lower the impulse inflicted on the specimen, the lesser number of fringes appeared on the interferograms for the same amount of thermal excitation. This would suggest a turn key solution in determining as well the extent of damage. Some of the specimens were cross sectioned and examined. This revealed that the core had been crushed under the skin in the impact region but the shape had regained perhaps due to the flexural 
strength of the skin. However, when slightly loaded it was noticed that the core easily compressed and the specimen was a great deal weaker in the impact region. The lack of delamination at the skin-core interface does not agree with previous held conviction. What may in fact be happening is that an imperceptible dent has occurred and when the specimen is thermally loaded the skin flexes outwards against the weakened or crushed core much in the same way as if a delamination existed. The results presented in figures 5, 6, 7 and 8 clearly demonstrate that all three techniques, that is, IRT, Shearography and ESPI, detect and show readily the location of defects in the composite material tested. Moreover, the IRT images were not only indicating the position of the defect or flaw, but produced data concerning the temperature of the region of the defect in relation to the cooler surrounding material. The Shearographic system however, was exceptional in the display of the results. The system was not just more sensitive compared to IRT in responding to the heating stimulus on the specimens and readily displayed the image denoting the defect, but was also able to show the image for much longer period of time than the IRT system. In all the test results obtained, both the Shearographic and ESPI systems continued to show live fringes while the IRT system had lost the 'hot spot'. This effect can be explained quite simply; as the specimen's surface cools it is very probable that below the skin the temperature around the defect is still higher then equilibrium thus straining the body which obviously affects or strains the skin surface as well, hence the surface is still strained although it's temperature has reached equilibrium with the environment.

\section{References}

1. J. Gryzagoridis, D. Findeis and W. Bopape "Impact damage detection on composites using Electronic Speckle Pattern Interferometry" Commadem 2005

2. D Galela, "FAA Inspection Research Activities for Composite Materials" The 2006 Composite Damage Tolerance \& Maintenance Workshop, July 20, 2006

3. Y Y Hung, "A speckle-shearing interferometer: a tool for measuring derivatives of surface displacement” Opt. Commun. 11 (2) (1974) 132-135.

4. Y Y Hung, "Shearography: a new optical method for strain measurement and nondestructive testing" Opt.Eng.21 (3) (1982) 391-395.

5. J Gryzagoridis, D Findeis "Benchmarking Shearographic NDT for Composites" Insight May 2008, Vol. 50 Issue 5, p249-252

6. J Butters and J Leendertz "Speckle pattern and holographic techniques in engineering metrology" Opt. Laser Technol. 3, 26-30, (1971)

7. J Gryzagoridis and D Findeis "Simultaneous Shearographic and Thermographic NDT of Aerospace materials" Insight, May2006, Vol. 48 Issue 5, p294-297

8. J Gryzagoridis and D Findeis "ESPI of Aerospace Composites Using Digital Shearography" Proceedings of the International Symposium on NDT in Aerospace, December 3-5, 2008, Fürth, Germany 